\title{
Validation Process for LEWICE by Use of a Naviér-Stokes Solver
}

\author{
William B. Wright \\ Vantage Partners, LLC \\ Christopher E. Porter \\ NASA Glenn Research Center
}

June 6, 2017

\section{Glenn Research Center}




\section{Outline}

- Validation History

- LEWICE-Pointwise-FUN3D Script

- Validation Process

- Results 


\section{Validation History}

- Many icing Codes Use a Small Set of Cases for Validation

- Typical Shape (below) from Tunnel Calibration Test

- 46 tracings available

- Warm Glaze Shows Wide Variation in Experiment Shapes

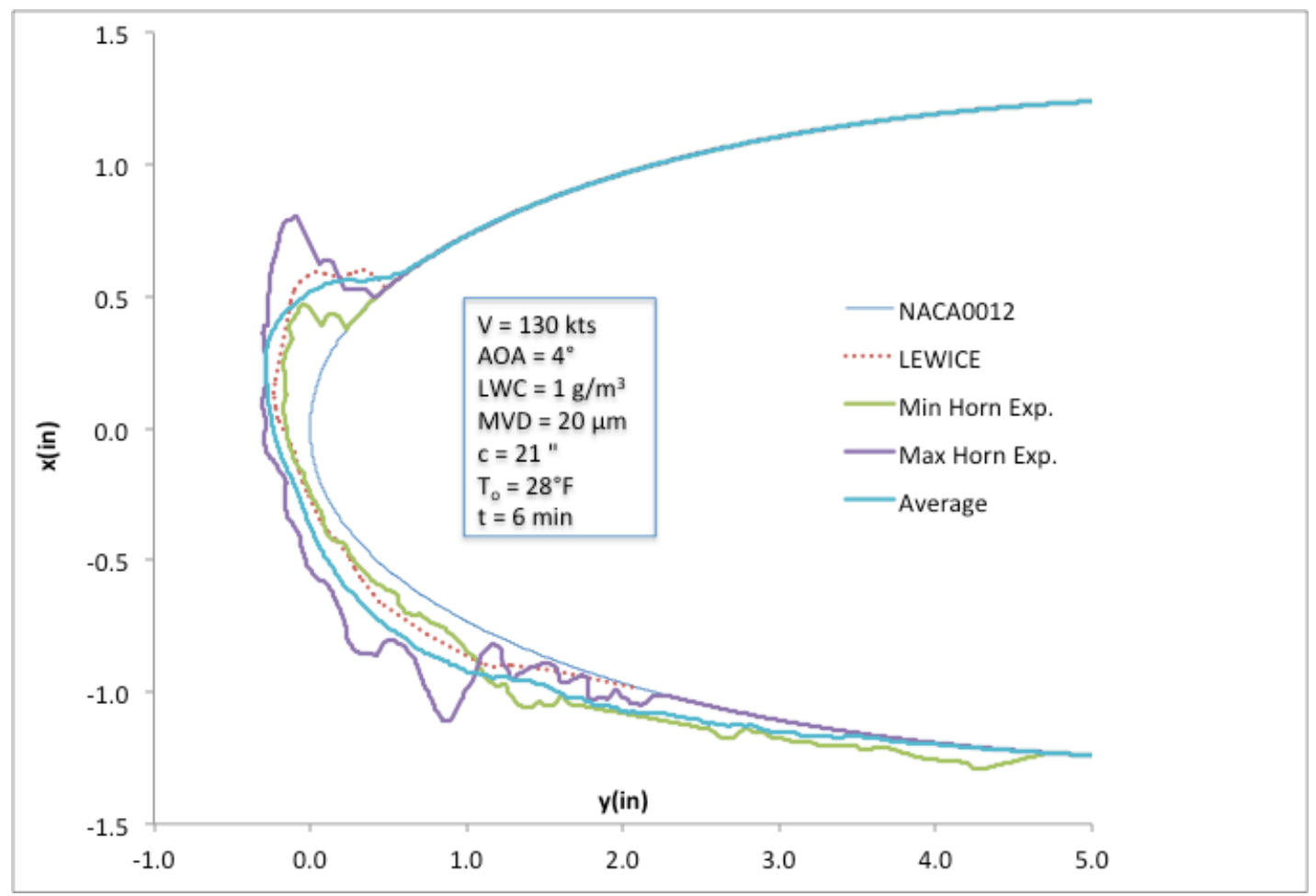

\section{Glenn Research Center}




\section{Geometric Validation}

- Previous LEWICE Validations Were Limited to Geometry Comparison (THICK Utility)

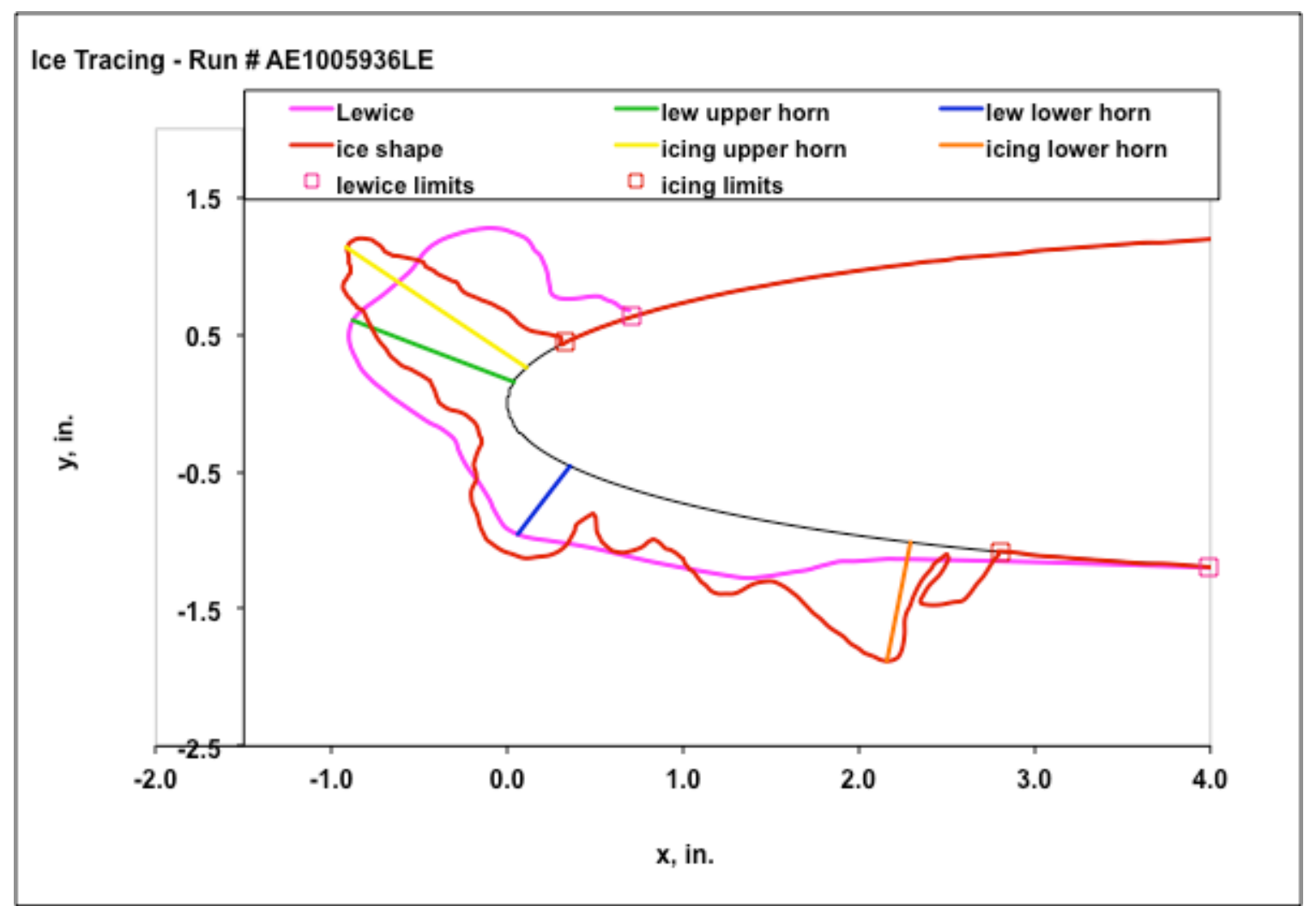

\section{Glenn Research Center}




\section{Revised Validation}

- Perform Geometric Comparisons as Before

- Perform Naviér-Stokes Calculations On Tracings from Experiment and LEWICE Predictions

- 12 conditions (all large glaze ice shapes)

- Compare lift and drag (AOA from $-6^{\circ}$ to $8^{\circ}$ )

- Three Conditions have Experimental Aero Data

- Compare CFD to experiment

- Show variation in aero performance

- Repeat shapes

- Variation of shape along span

- Smoothing

- Solutions performed with NASA FUN3D

- Wilcox 2006 turbulence model 


\section{Conditions}

\begin{tabular}{|c|c|c|c|c|c|c|c|c|c|}
\hline Airfoil & Chord (m) & $\begin{array}{l}\text { Air Speed } \\
(\mathrm{m} / \mathrm{s})\end{array}$ & $\mathrm{AOA}(\mathrm{deg})$ & $\begin{array}{c}\text { Total Temp } \\
\left(\begin{array}{ll}{ }^{\circ} & \mathrm{C}\end{array}\right)\end{array}$ & $\begin{array}{l}\text { LWC } \\
\left(\mathrm{g} / \mathrm{m}^{3}\right)\end{array}$ & $\operatorname{MVD}(\mu \mathrm{m})$ & Time (s) & Mach & $\mathrm{Re}^{*} 10-6$ \\
\hline NACA0012 & 0.53 & 102.9 & 4 & -5.6 & 1.8 & 30 & 360 & 0.32 & 4.40 \\
\hline NACA0012 & 0.91 & 77.1 & 0 & -8.3 & 1 & 15 & 1224 & 0.24 & 5.67 \\
\hline NACA23012 & 0.46 & 102.9 & 2 & -2.2 & 0.75 & 15.4 & 300 & 0.31 & 3.68 \\
\hline NACA23012 & 1.83 & 90.0 & 2 & -5.0 & 0.94 & 15 & 600 & 0.28 & 13.03 \\
\hline BizJet & 0.30 & 139.4 & 0 & -7.9 & 1.12 & 19 & 138 & 0.44 & 3.58 \\
\hline BizJet & 0.46 & 88.4 & 0 & -6.1 & 1.47 & 29 & 240 & 0.27 & 3.22 \\
\hline BizJet & 0.61 & 89.4 & 0 & -6.0 & 1.3 & 35 & 360 & 0.27 & 4.34 \\
\hline BizJet & 0.61 & 89.4 & 0 & -13.0 & 1.27 & 43 & 360 & 0.28 & 4.57 \\
\hline BizJet & 0.91 & 90.0 & 6 & -0.8 & 0.54 & 20 & 1350 & 0.27 & 6.32 \\
\hline NLF0414 & 0.91 & 66.9 & 2 & -3.1 & 0.54 & 20 & 1350 & 0.20 & 4.71 \\
\hline LTHS & 0.91 & 130.2 & 0 & -1.4 & 0.563 & 21 & 1476 & 0.40 & 9.47 \\
\hline NACA0015 & 0.35 & 95.2 & 0 & -5.5 & 0.75 & 19 & 600 & 0.29 & 2.68 \\
\hline
\end{tabular}




\section{Sample Grid}

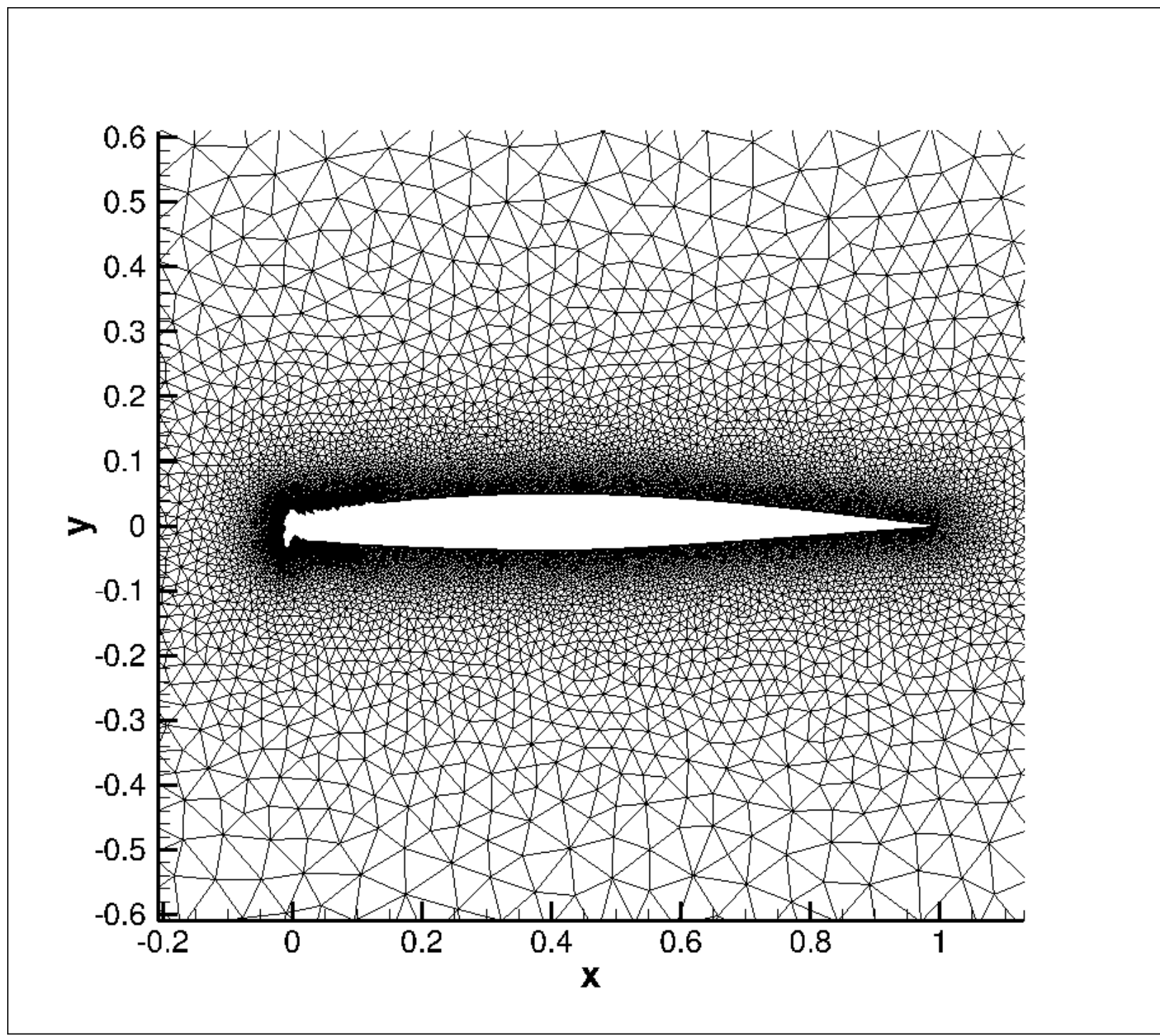

\section{Glenn Research Center}




\section{Sample Grid}

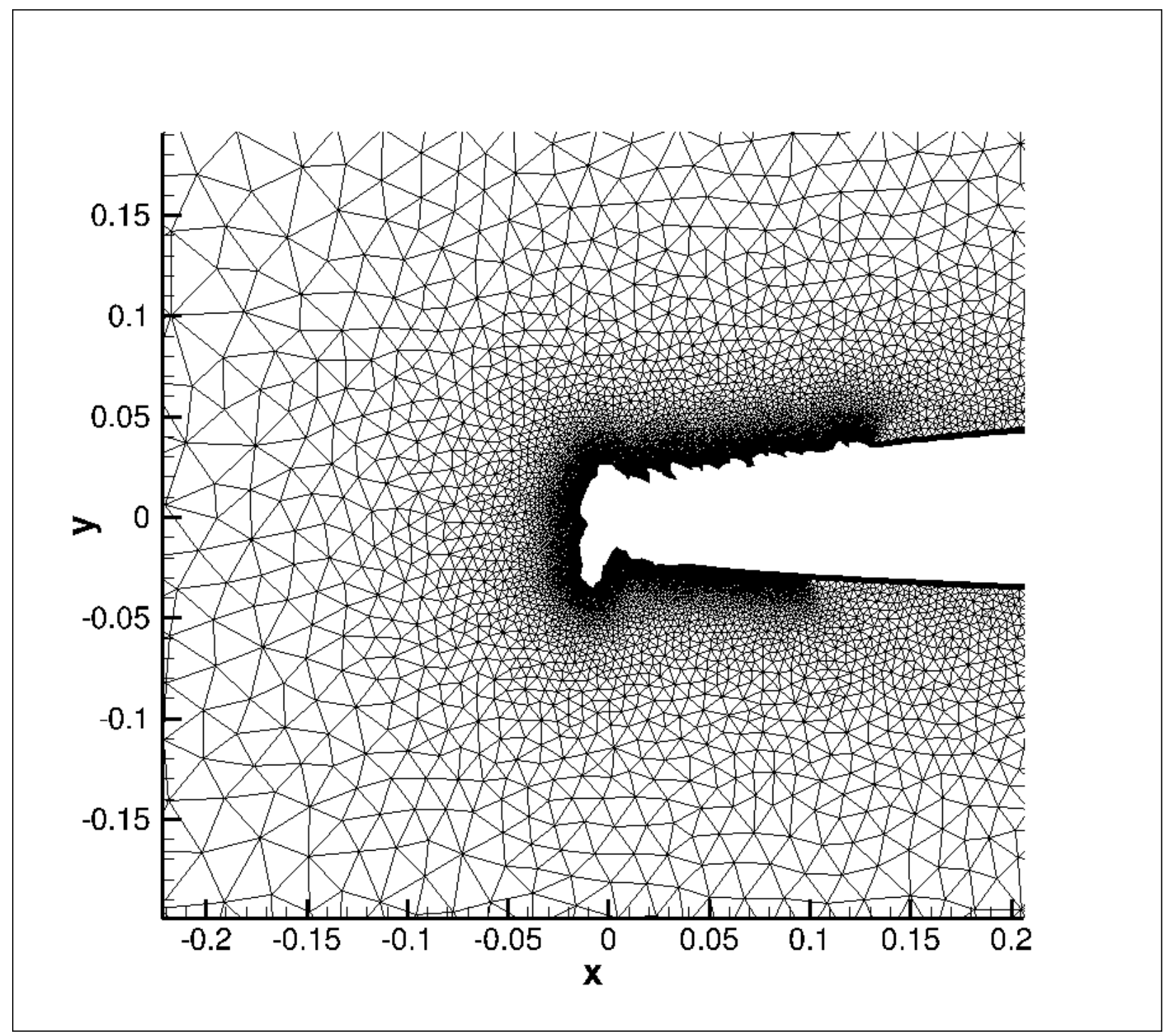

\section{Glenn Research Center}




\section{Validation Results}

- Geometric Differences between LEWICE and Experiment Less Than Experimental Variability of Full Database

- Geometric Differences of Experimental Shapes Chosen Relatively Small 


\section{HF1009536 Aero Comparison}
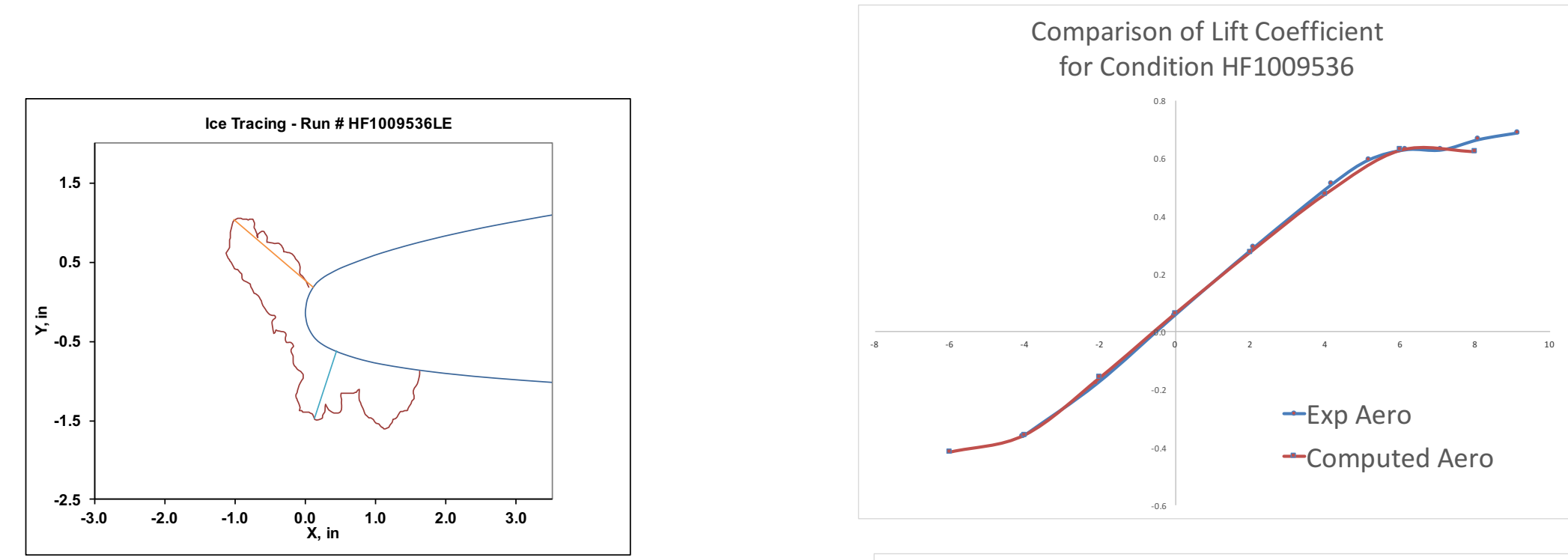

Glenn Research Center

Comparison of Computed Drag Coefficient

for Condition HF1009536

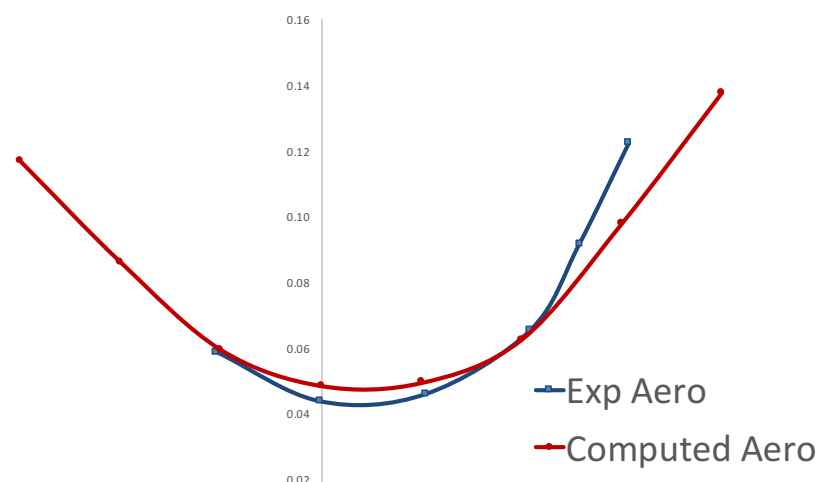




\section{HF1009536}

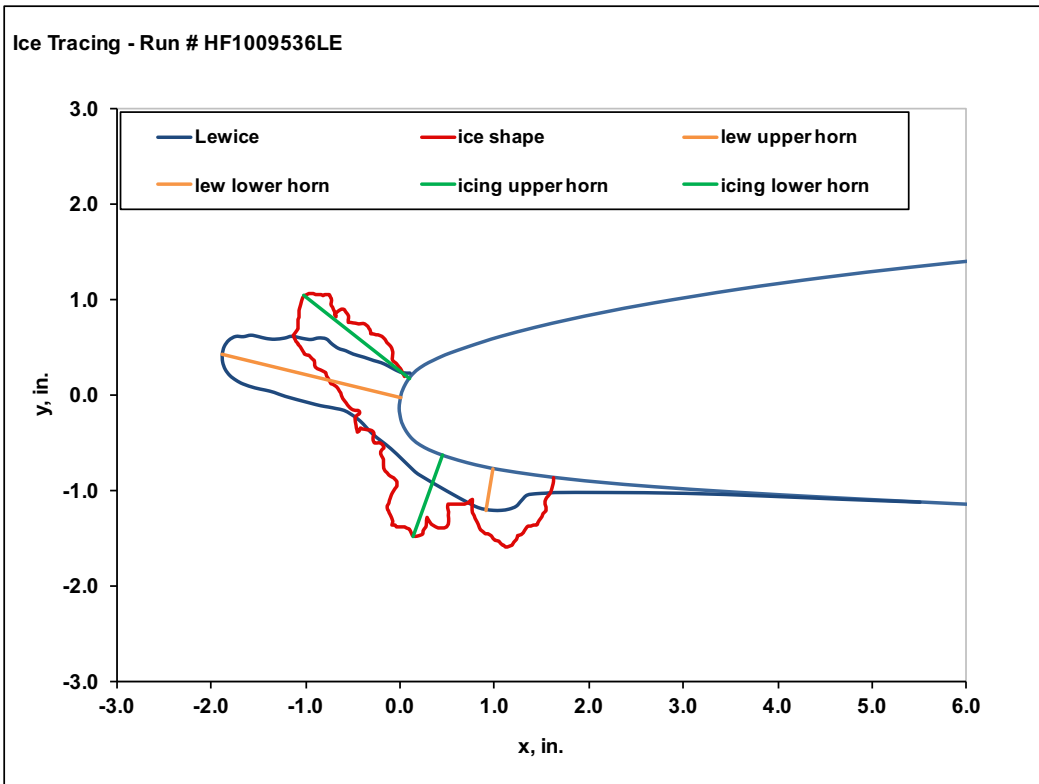

Comparison of Computed Lift Coefficient

for Condition HF1009536
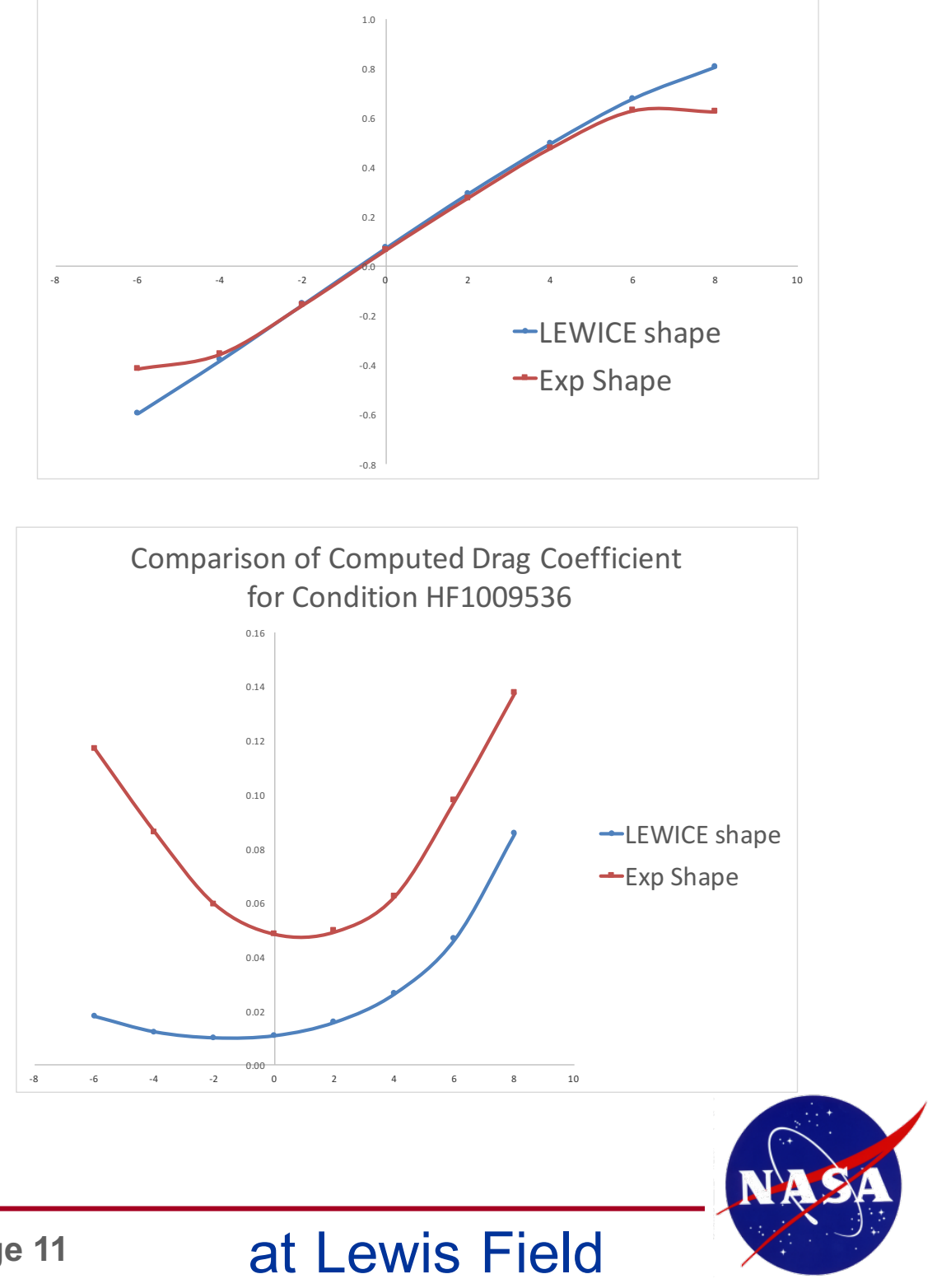

Glenn Research Center 


\section{HF1009536 Repeatability}
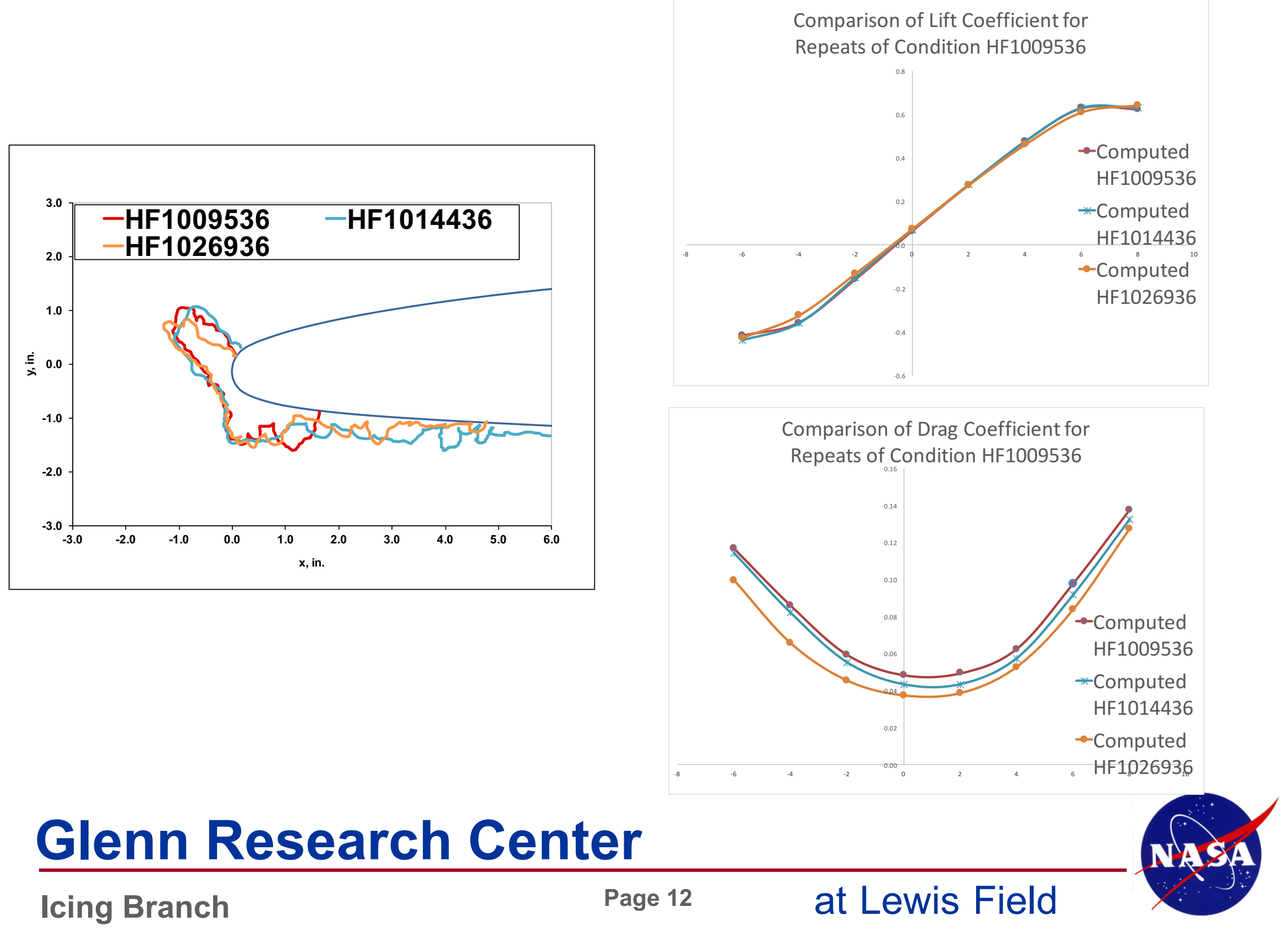

\section{Glenn Research Center}




\section{ED073536 Aero Comparison}
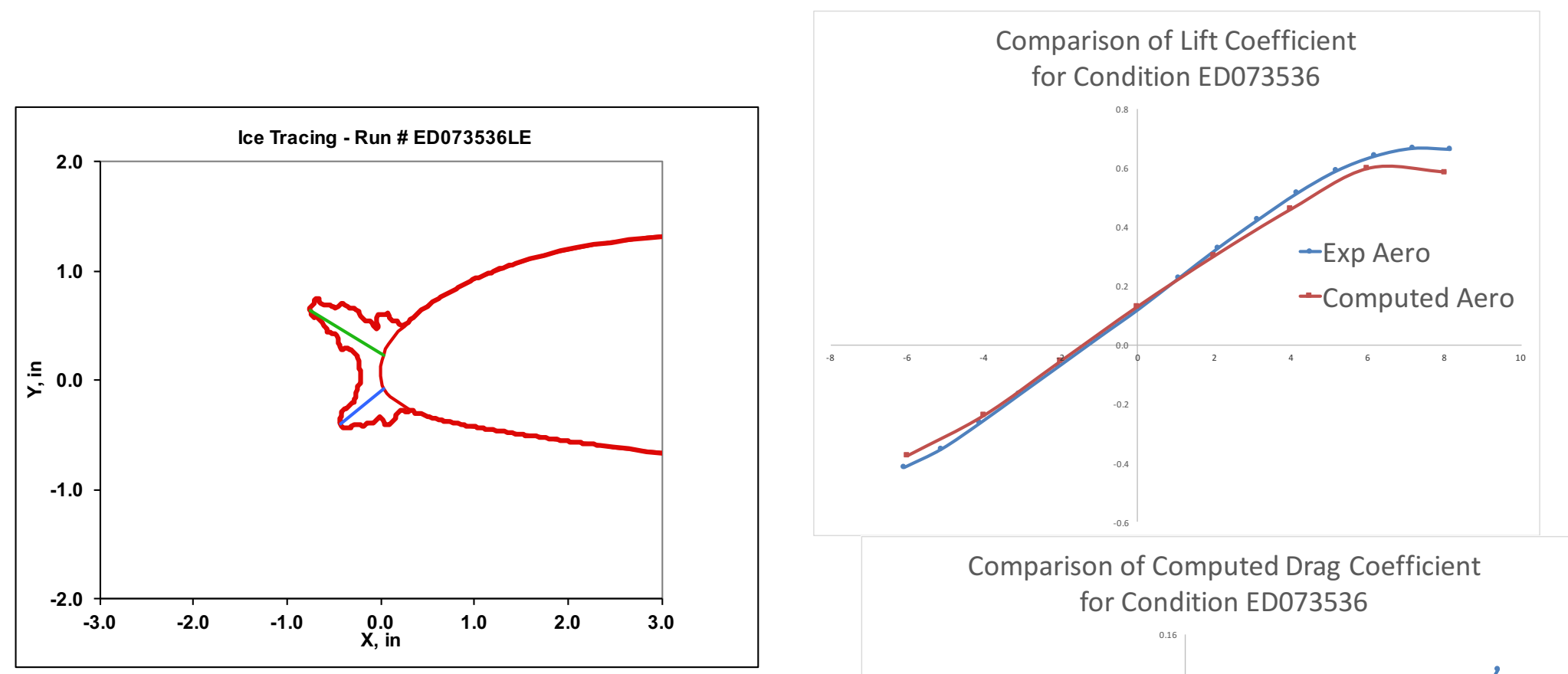

Comparison of Computed Drag Coefficient for Condition ED073536

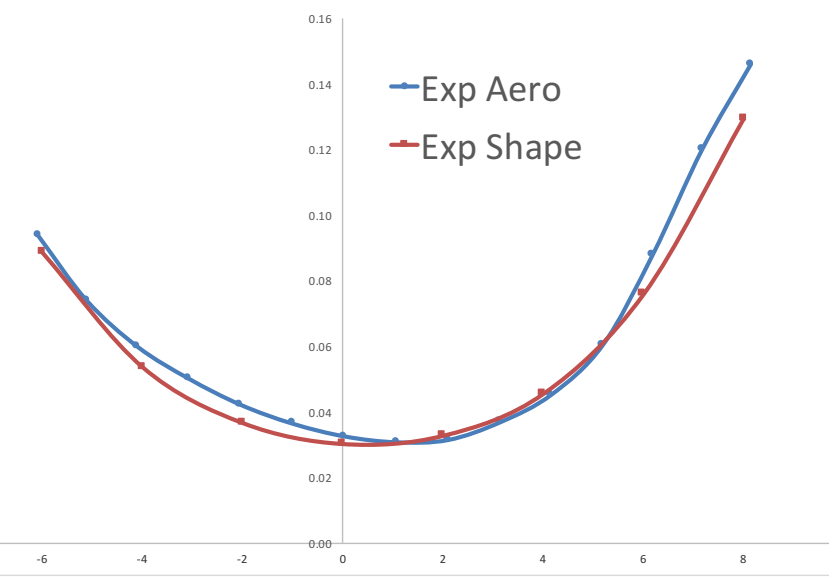

\section{Glenn Research Center}




\section{ED073536}
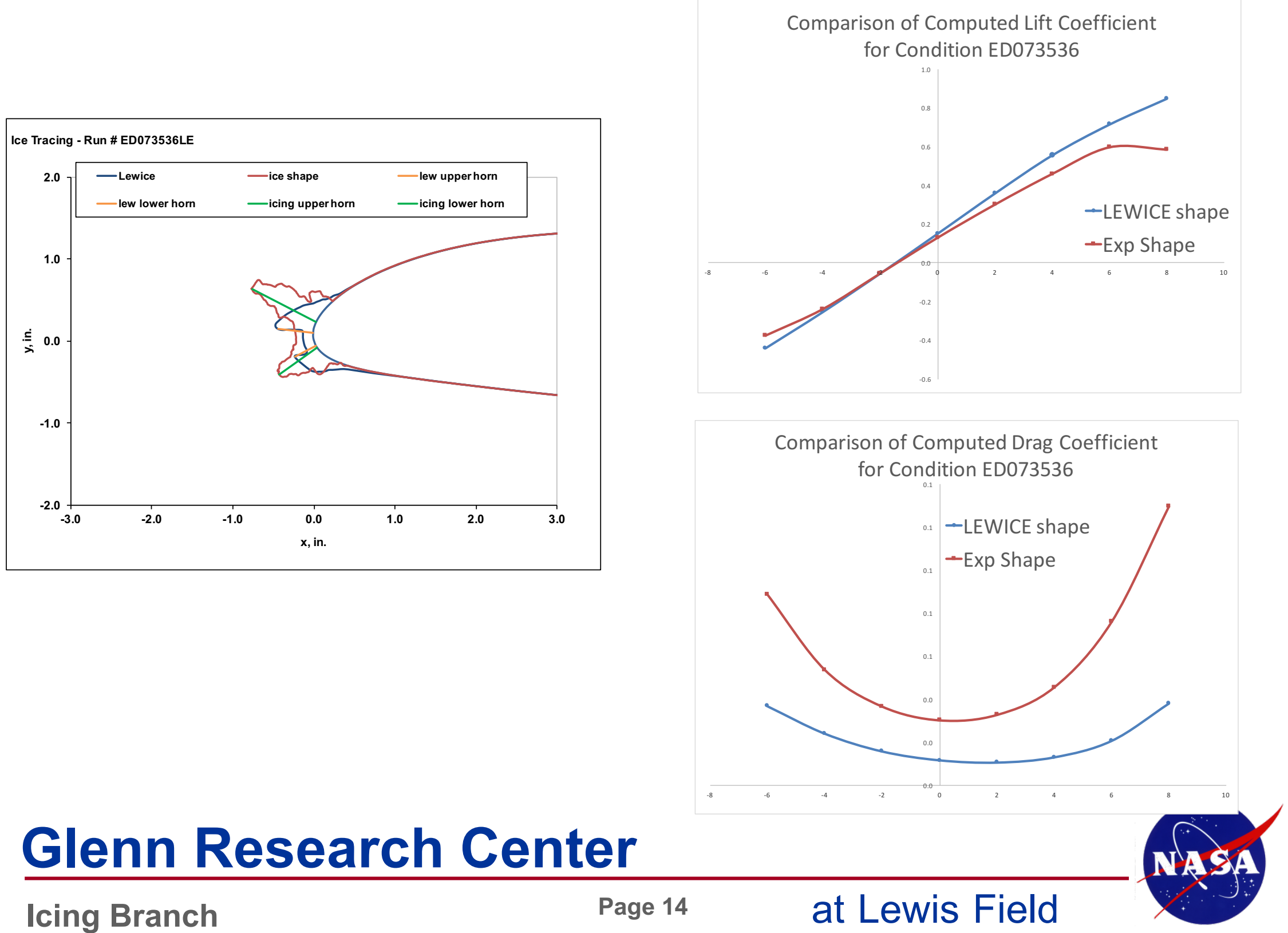

Glenn Research Center 


\section{ED073536 Repeatability}
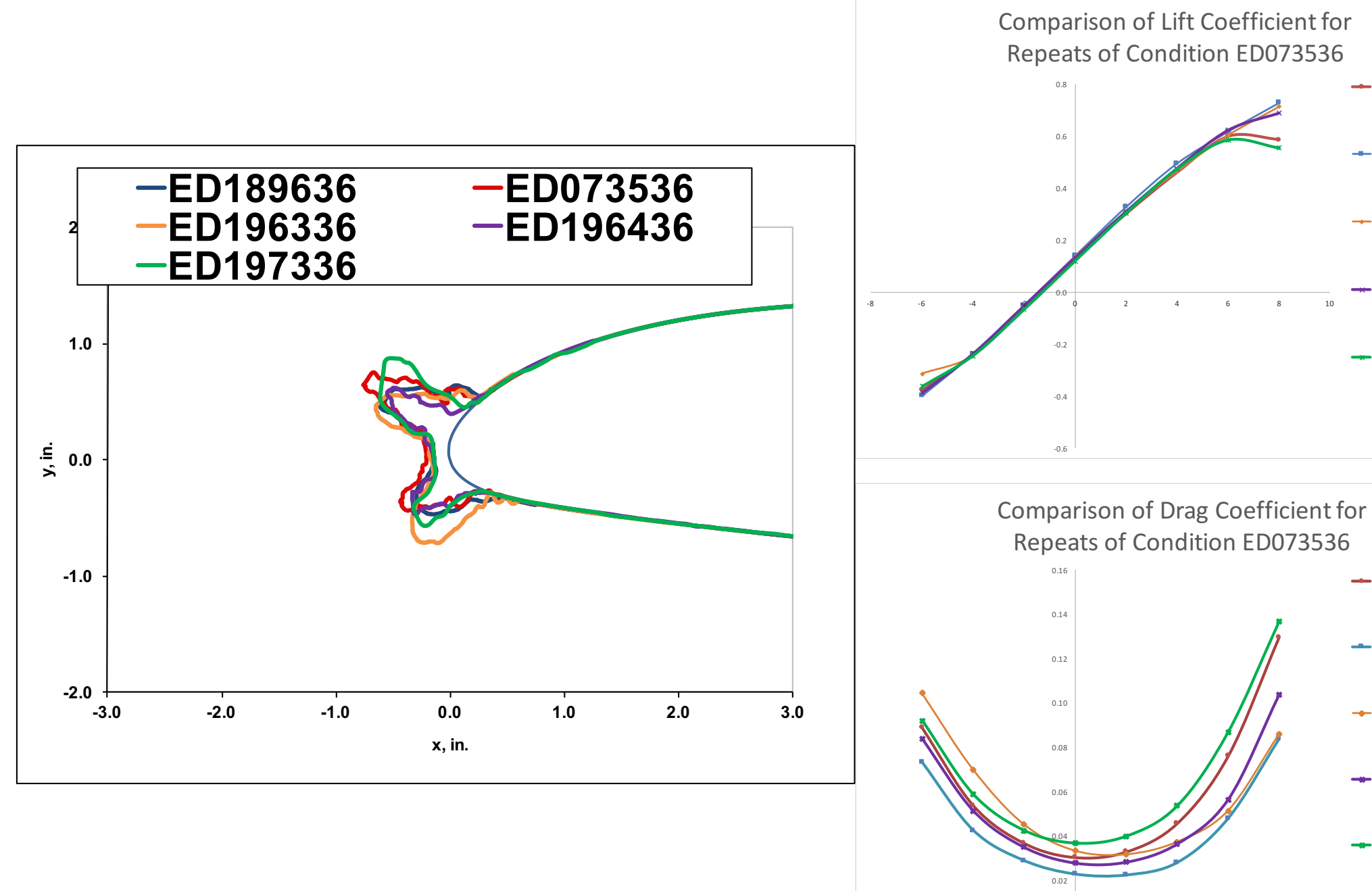

-Computed ED073536

-Computed ED189636

-Computed ED196336

-Computed ED196436

-Computed ED197336

\section{Glenn Research Center}




\section{AF1181836}
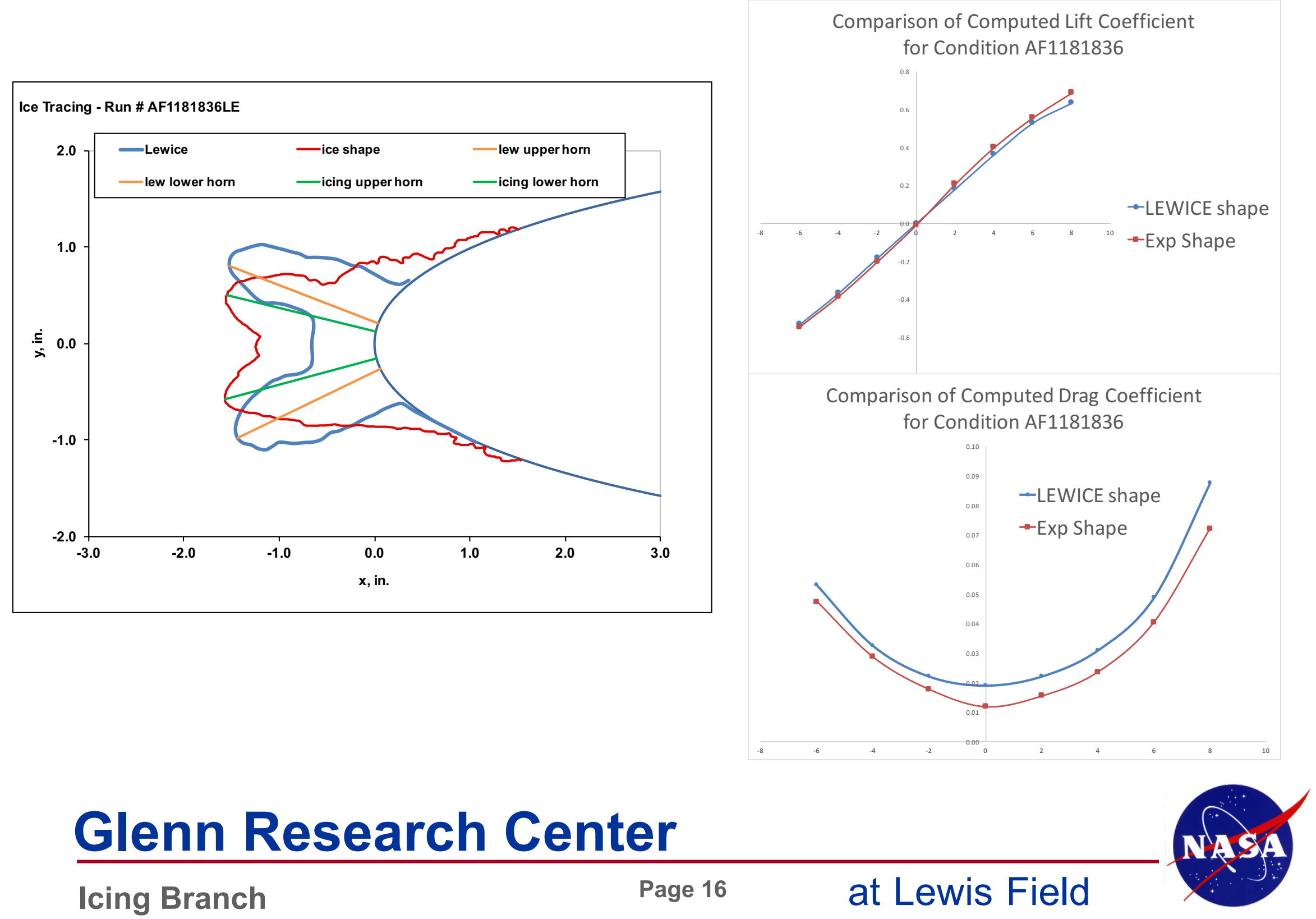

Glenn Research Center 


\section{HE1080536}
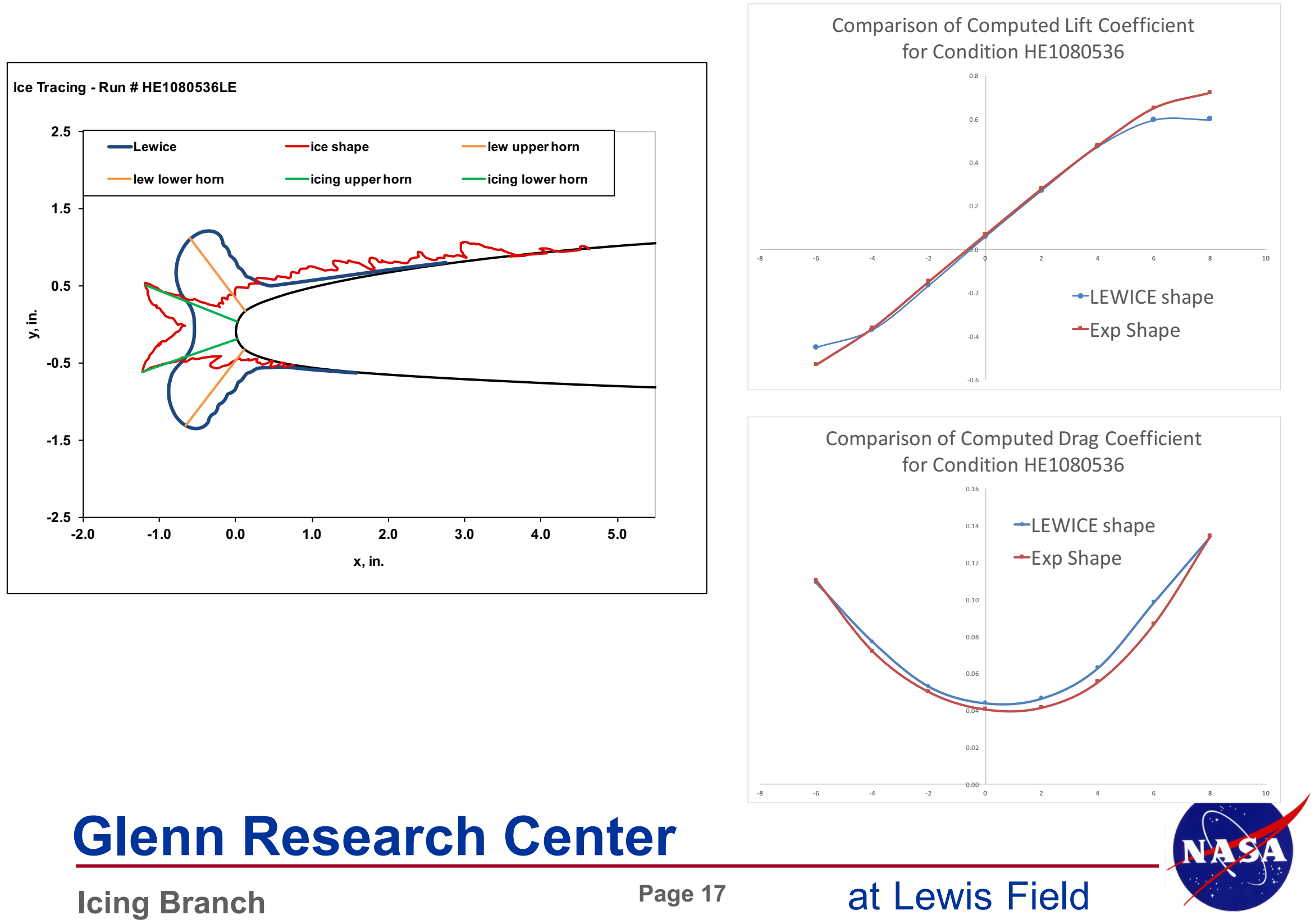

\section{Glenn Research Center}




\section{HD1075636}

Ice Tracing - Run \# HD1075636LE

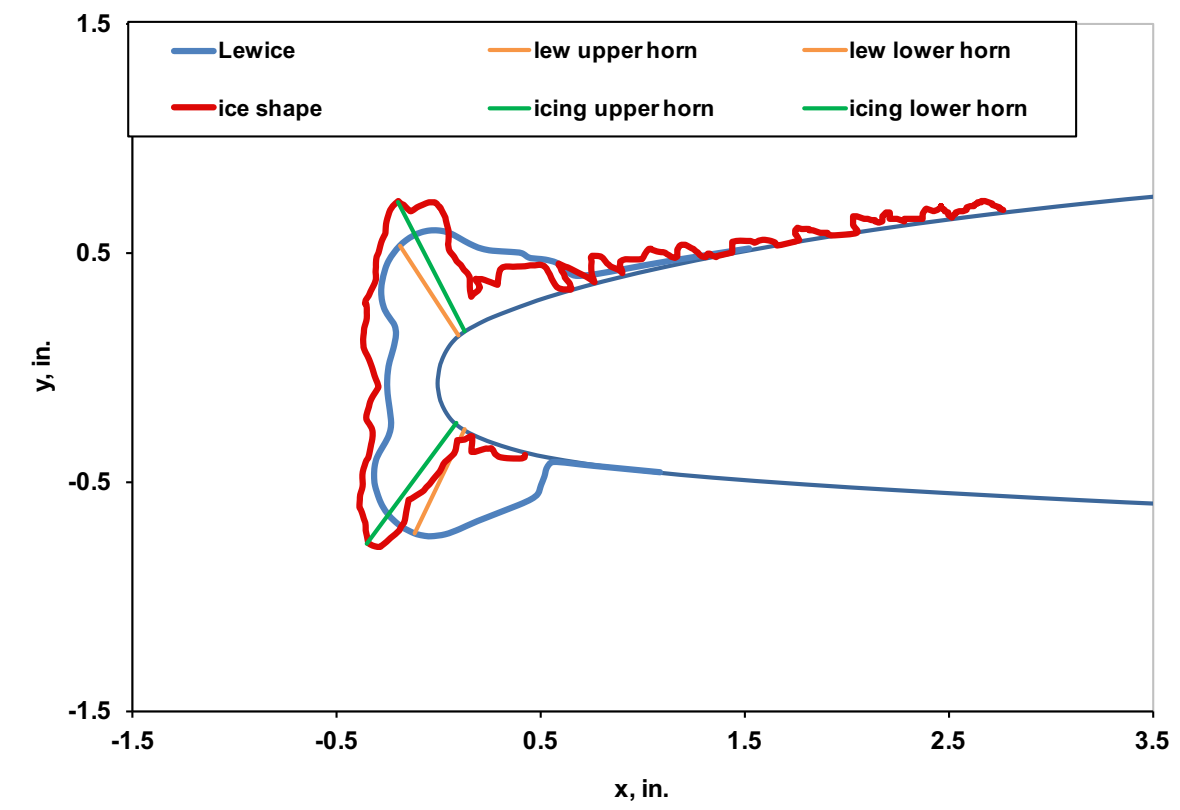

\section{Glenn Research Center}

-LEWICE shape

-Exp Shape 


\section{Mach Contours for HD1075636}

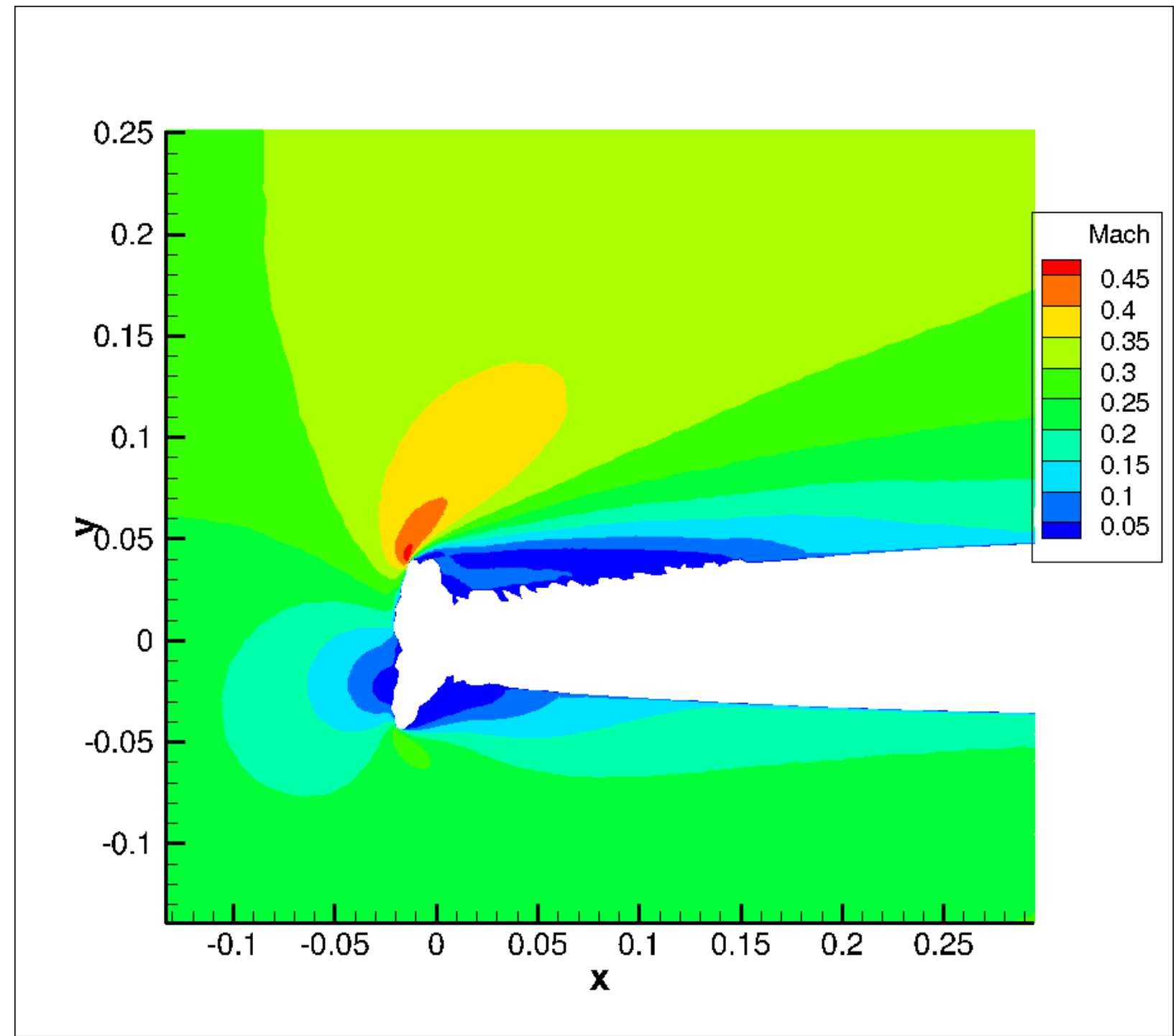

\section{Glenn Research Center}




\section{Mach Contours for HD1075736}

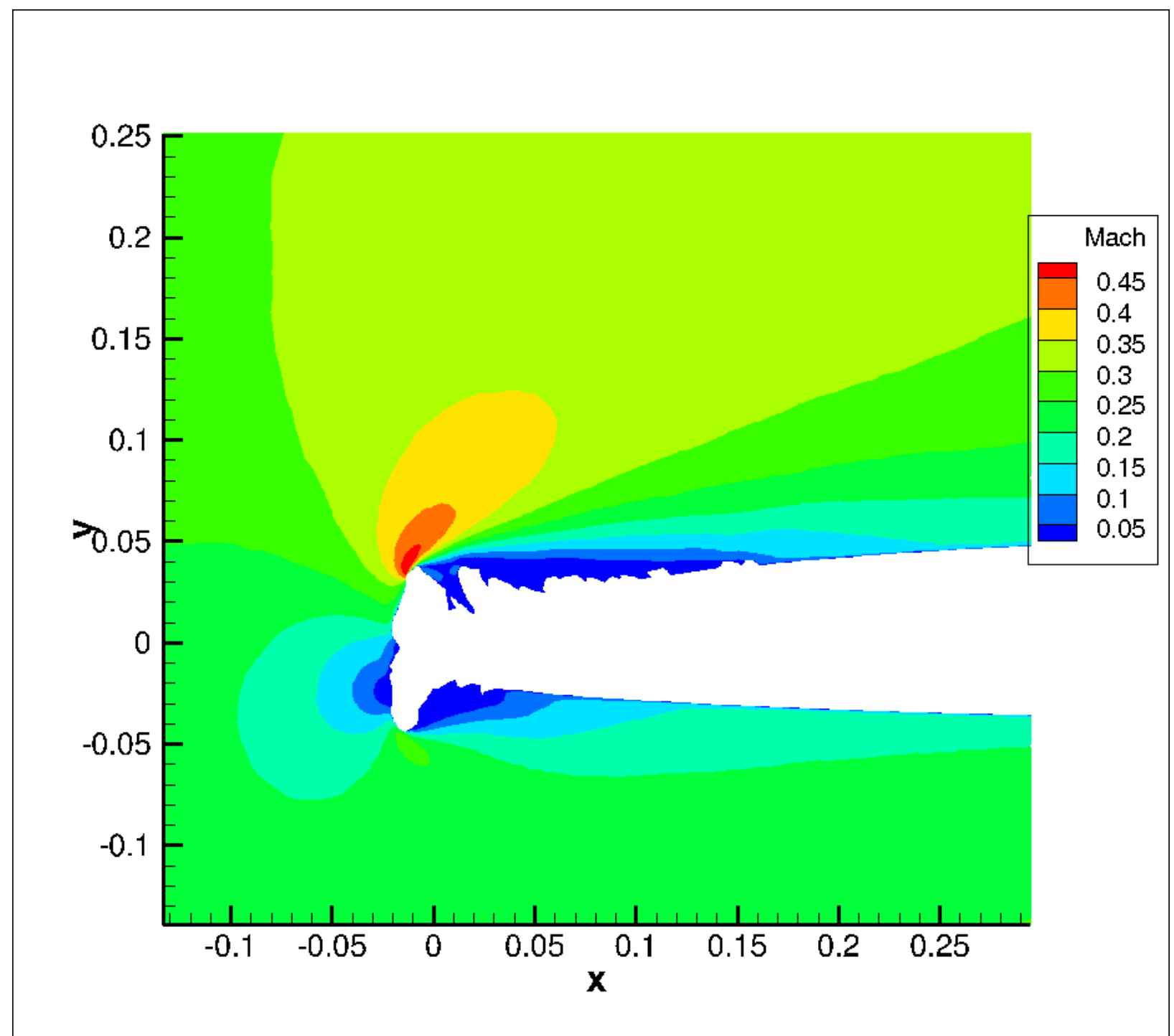

\section{Glenn Research Center}




\section{Mach Contours for HD1075936}

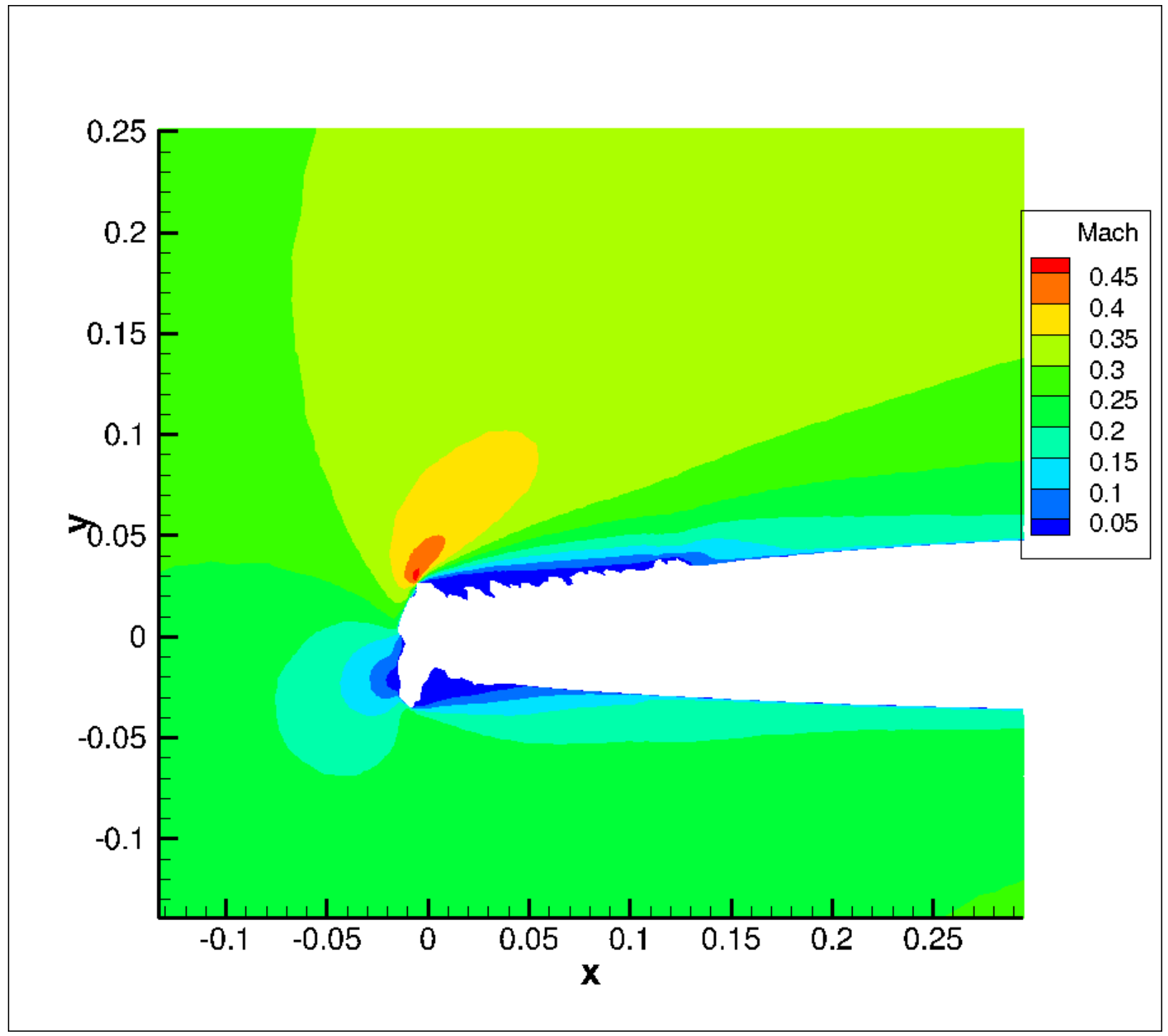

\section{Glenn Research Center}




\section{Mach Contours for Corresponding LEWICE Shape}

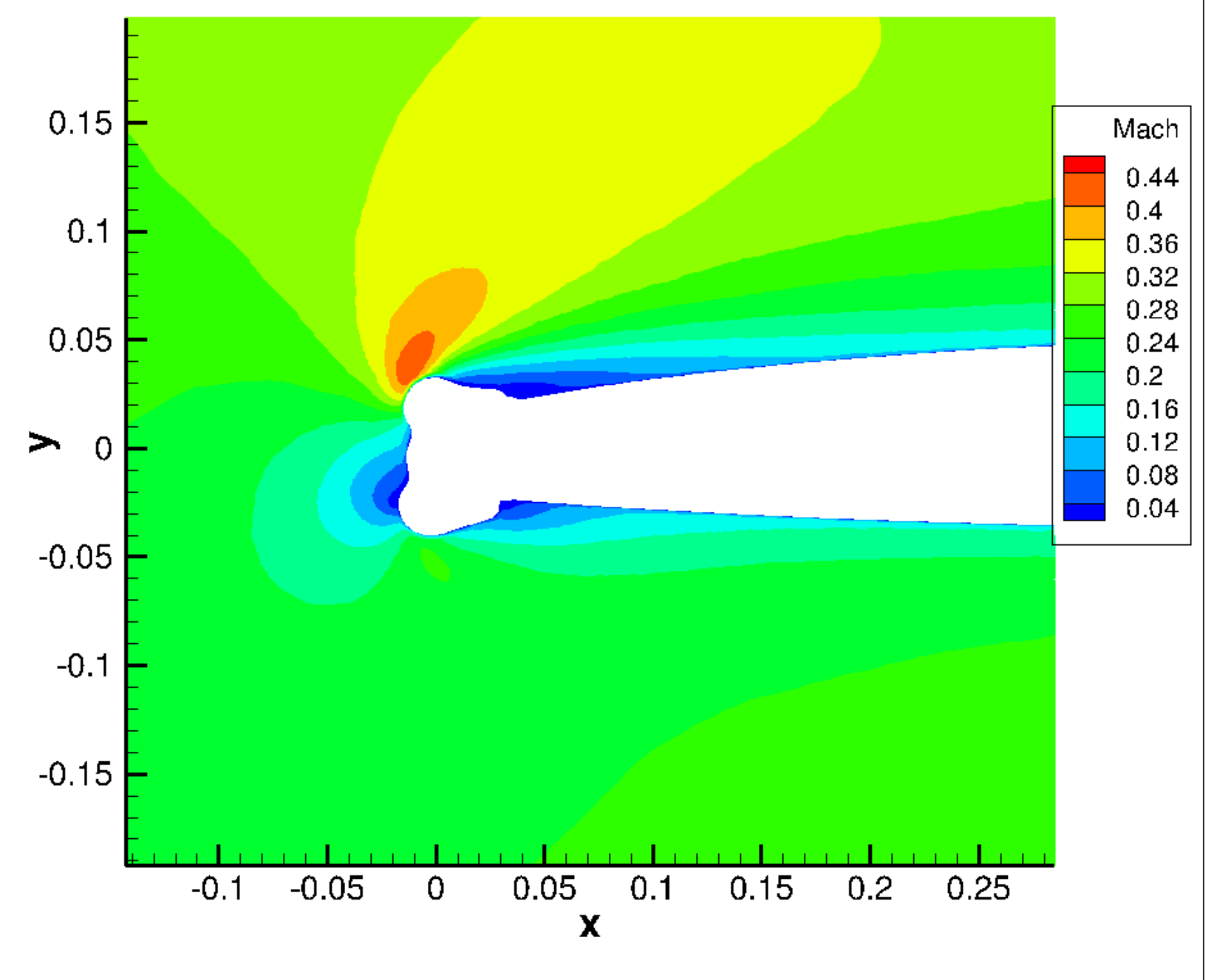

\section{Glenn Research Center}




\section{Conclusions}

- LEWICE model updated to include additional grid options

- New python script allows user to run hundreds of cases with or without Naviér-Stokes

- Geometric comparison shows good agreement with Experiment

- This study suggests that geometrically similar shapes can produce different performance characteristics

- Lift and drag calculations of ice shapes show favorable agreement with experiment

Glenn Research Center 